\title{
Clustering Coefficients of Protein-Protein Interaction Networks
}

\author{
Gerald A. Miller*, Yi Y. Shi ${ }^{\dagger}$, Hong Qian ${ }^{\dagger}$, and Karol Bomsztyk ${ }^{\ddagger}$ \\ Departments of ${ }^{*}$ Physics, ${ }^{\dagger}$ Applied Mathematics, and ${ }^{\ddagger}$ Medicine, \\ University of Washington Seattle, WA 98195
}

\begin{abstract}
The properties of certain networks are determined by hidden variables that are not explicitly measured. The conditional probability (propagator) that a vertex with a given value of the hidden variable is connected to $\mathrm{k}$ of other vertices determines all measurable properties. We study hidden variable models and find an averaging approximation that enables us to obtain a general analytical result for the propagator. Analytic results showing the validity of the approximation are obtained. We apply hidden variable models to protein-protein interaction networks (PINs) in which the hidden variable is the association free-energy, determined by distributions that depend on biochemistry and evolution. We compute degree distributions as well as clustering coefficients of several PINs of different species; good agreement with measured data is obtained. For the human interactome two different parameter sets give the same degree distributions, but the computed clustering coefficients differ by a factor of about two. This shows that degree distributions are not sufficient to determine the properties of PINs.
\end{abstract}




\section{INTRODUCTION}

Physicists have recently shown that network analysis is a powerful tool to study the statistical properties of complex biological, technological and social systems of diverse kinds [1, 2, 3]. Many networks exhibit a scale-free degree distribution in which the probability $p_{k}$ that a vertex is connected to $k$ other vertices falls as a power $p_{k} \sim k^{-\gamma}$. This property is not sufficient to completely describe natural networks because such systems also exhibit degree correlationsthe degrees of the vertices at the end points of any given edge are not independent [4, 5, 6, 7]. It is not surprising that natural systems depend on properties that do not appear explicitly in degree distributions. In particular, protein interaction networks depend on the availability of sufficient binding free energy [8] to cause interactions to occur (links between vertices to exist).

Caldarelli et al. [9] and Söderberg [10] proposed models in which vertices are characterized by a fitness parameter assigned according to a chosen probability distribution. Then, pairs of vertices are independently joined by an undirected edge with a probability depending on the fitnesses of the end points. Ref. [11] generalized these models as a class of models with hidden variables and presented a detailed formalism showing how to compute network properties using the conditional probability (propagator) that a vertex with a given value of a hidden variable is connected to other $k$ vertices. This formalism, valid for any Markovian (binary) network, provides the generating function for the propagator, but not the propagator itself.

The purpose of this paper is twofold. We first use a mean field approximation to derive a general analytic formula for the propagator, therefore finding a general approximate solution to to the inversion problem. This enables one to compute network properties without the use of a simulation procedure, thereby simplifying the computational procedure and potentially broadening the ability of scientists from all fields to use network theory. The validity of the method is assessed by comparing the results of using our approximation with published results. We then use this method to compute clustering coefficients of a specific hidden variable model for protein-protein interaction networks (PIN) from several organisms developed by us[12] that previously had obtained degree distributions in agreement with measured data. We show that two models with the same degree distribution have very different clustering coefficients.

We outline this in more detail. Sect. II reviews the hidden variable formalism and our approximate solution to the inversion problem. We distinguish between sparse (which have been solved in Ref. [1] ) and non-sparse networks which are solved here. The next section III studies the models of Refs. [9] and [13]. Our averaging procedure is found to work well for most situations. Our own model[12] is presented in IV. We present an analytic result for the average connection probability and extend the results of [12] to computing the clustering coefficients. The final section $\mathrm{V}$ is reserved for a brief summary and discussion.

\section{HIDDEN VARIABLE NETWORKS}

We present the formalism for hidden variable models [11]. The probability that a node has a hidden continuous variable $g$ is given by $\rho(g)$, normalized so that its integral over its domain is unity. This function is chosen to be an exponential in [9, 12] and a Gaussian in [13]. The connection probability for two nodes of $g, g^{\prime}$ is defined to be $p\left(g, g^{\prime}\right)$. This is 
taken as a step function in [9, 13], and a Fermi function in [12]. The two functions $\rho(g)$ and $p\left(g, g^{\prime}\right)$ can be chosen in a wide variety of ways to capture the properties of a given network. Reference [11] presents the probability generating function, $G_{0}(x)$, that determines $p_{k}$ in terms of the generating function for the propagator, $\widehat{G}_{0}(z, g)$, as

$$
G_{0}(z)=\int d g \rho(g) \widehat{G}_{0}(z, g)
$$

where

$$
\ln \widehat{G}_{0}(z, g)=N \int d g^{\prime} \rho\left(g^{\prime}\right) \log \left(1-(1-z) p\left(g, g^{\prime}\right)\right)
$$

The propagator $G_{0}(k, g)$ giving the conditional probability that a vertex of hidden variable $g$ is connected to $k$ other vertices is given implicitly by

$$
\widehat{G}_{0}(z, g)=\sum_{k=0}^{\infty} z^{k} G_{0}(k, g) .
$$

Knowledge of $G_{0}(k, g)$ determines the conditional probability $P\left(k^{\prime} \mid k\right)$ that a node of degree $k$ is connected to a node of degree $k^{\prime}$, [11] (as well as $p_{k}$ ), and those two functions completely define a Markovian network. Once $G_{0}(k, g)$ is the determined, all of the properties of the given network are determined. The most well-known example is the degree distribution $p_{k}$ :

$$
p_{k}=\int_{0}^{\infty} d g \rho_{\lambda}(g) G_{0}(k, g)
$$

It would seem that determining $G_{0}(k, g)$ from Eq. (2) is a simple technical matter, but this is not the case[11]. The purpose of the present Section is to provide a simple, analytic and accurate method to determine $G_{0}(k, g)$.

We obtain $G_{0}(k, g)$ from Eq. (2) by using the tautology

$$
p\left(g, g^{\prime}\right)=\bar{p}(g)+\left(p\left(g, g^{\prime}\right)-\bar{p}(g)\right)
$$

in Eq. (2), choosing $\bar{p}(g)$ so as to eliminate the effects of the second term, and then treating the remaining higher powers of $\left(p\left(g, g^{\prime}\right)-\bar{p}(g)\right)$ as an expansion parameter. Using Eq. (5) in Eq. (2) yields

$$
\begin{aligned}
& \ln \widehat{G}_{0}(z, g)=\ln \widehat{G}_{0}(z, g)=\log (1-(1-z) \bar{p}(g))^{N}-N(1-z) \int d g^{\prime} \rho\left(g^{\prime}\right) \frac{\left(\bar{p}(g)-p\left(g, g^{\prime}\right)\right)}{1-(1-z) \bar{p}(g)} \\
& -N \sum_{n=2}^{\infty} \frac{(1-z)^{n}}{n} \int d g^{\prime} \rho\left(g^{\prime}\right)\left(\frac{\left.p\left(g, g^{\prime}\right)-\bar{p}(g)\right)}{1-(1-z) \bar{p}(g)}\right)^{n} .
\end{aligned}
$$

In analogy with the mean-field (Hartree) approximation of atomic and nuclear physics, we find that the second term of Eq. (6) vanishes if we choose $\bar{p}(g)$ to be the average of $p\left(g, g^{\prime}\right)$ over $\rho\left(g^{\prime}\right)$ :

$$
\bar{p}(g)=\int d g^{\prime} \rho\left(g^{\prime}\right) p\left(g, g^{\prime}\right)
$$


With Eq. (7) the effects of the term of first order in $\left(p\left(g, g^{\prime}\right)-\bar{p}(g)\right)$ vanish. We therefore obtain the result:

$$
\ln \widehat{G}_{0}(z, g)=\log (1-(1-z) \bar{p}(g))^{N}-N \sum_{n=2}^{\infty} \frac{(1-z)^{n}}{n} \int d g^{\prime} \rho\left(g^{\prime}\right)\left(\frac{\left.p\left(g, g^{\prime}\right)-\bar{p}(g)\right)}{1-(1-z) \bar{p}(g)}\right)^{n},
$$

with the putative term with $n=1$ vanishing by virtue of Eq. (7).

We treat the first term of Eq. (8) as the leading order $(L O)$ term and regard the remainder as a correction. The validity of this approach can be checked by comparison with simulations, or (in certain cases) with analytic results. Numerical results for the PIN of current interest [12] indicate that the corrections to the $L O$ terms induce errors in $p_{k}$ of no more than a few percent and that the approximation becomes more accurate for large values of $k$. Therefore we use the $L O$ approximation. Using exponentiation and the binomial theorem in the first term of Eq. (8) leads to the result

$$
G_{0}^{(L O)}(k, g)=\left(\begin{array}{c}
N \\
k
\end{array}\right)(1-\bar{p}(g))^{N-k} \bar{p}(g)^{k}
$$

which is of the form of a random binomial distribution in which the connection probability depends on the hidden variable $g$. The Eq. (9) is our central new general result that can be used for any hidden variable network. This binomial distribution has both the normal Gaussian and Poisson $(N p(g) \ll 1)$ distributions as limiting cases.

\section{A. Sparse and Nonsparse Networks}

Ref. [11] explained the difference between sparse and nonsparse networks. Sparse networks have a well-defined thermodynamic limit for the average degree, while this quantity diverges as the network size $N$ approaches infinity. Ref. [11] defines criteria for sparseness by pointing out the relevance of $\bar{p}$ of Eq. (7) in determining whether or not a network is sparse. Given this quantity the average degree is

$$
\langle k\rangle=\int d g \rho(g) \bar{p}(g)=\int d g \int d g^{\prime} \rho(g) p\left(g, g^{\prime}\right) \rho\left(g^{\prime}\right) .
$$

If the $\rho(g)$ is independent of $N$ the only way to obtain a non-divergent value $\langle k\rangle$ is for the connection probability [11] to scale as $N^{-1}$ :

$$
p^{\text {sparse }}\left(g, g^{\prime}\right)=\frac{C\left(g, g^{\prime}\right)}{N}, \text { sparse network . }
$$

Under the specific assumption that Eq. (11) holds, Ref. [11] finds a very interesting result. In our notation, this amounts to using Eq. (11) in Eq. (2) and taking the limit that $N$ approaches infinity. Then

$$
G_{0}^{\mathrm{sparse}}(z, g)=\exp (z-1) \int d g^{\prime} \rho\left(g^{\prime}\right) C\left(g, g^{\prime}\right) \text {. }
$$

This shows that the Poisson limit of Eq. (9) is obtained for the very special case of sparse networks in which the connection probability scales as $N^{-1}$. None of the models of interest here [9, 12, 13] are sparse, so it is our present result (9) that is widely applicable. 


\section{B. General Networks}

Turning to the use of the use of the propagator, we obtain the degree distribution as

$$
p_{k}=\int d g \rho(g) G_{0}(k, g) \approx \int d g \rho(g) G_{0}^{(L O)}(k, g) .
$$

This expression can be thought of as averaging a binomial distribution over the hidden variable and is a natural generalization of classical graph theory. A similar expression for $p_{k}$ has been obtained, in the Poisson limit, in Ref. [15]. In that work, $p_{k}$ is presented as an integral of the Poisson distribution for $p(g)$ multiplied by the " $P$ representation" of a density matrix. Comparing Eq. (9) with the result (3) of [15] shows that our propagator is proportional to the $P$ representation, essentially our $\rho(g)$. Ref. [15] shows, how under certain assumptions, to use $p(k)$ to determine the $P$ representation. Our method allows underlying network properties, denoted by $\rho(g)$ and $p\left(g, g^{\prime}\right)$, to predict various network properties.

The clustering coefficient which measures transitivity [3]: if vertex $A$ is connected to vertex $B$ and vertex $B$ to vertex $C$, there is an increased probability that vertices $A$ and $C$ are connected. In graph theory, the clustering coefficient $c(k)$ is the ratio of the number of triangles to the number of pairs, computed for nodes of degree $k$. Ref. [11] shows that

$$
\begin{aligned}
& c(k)=\frac{1}{p_{k}} \int d g \rho(g) G_{0}(k, g) c(g) \\
& c(g)=\int d g^{\prime} \int d g^{\prime \prime} \frac{\rho\left(g^{\prime}\right) p\left(g, g^{\prime}\right)}{\bar{p}(g)} p\left(g^{\prime}, g^{\prime \prime}\right) \frac{\rho\left(g^{\prime \prime}\right) p\left(g^{\prime \prime}, g\right)}{\bar{p}(g)} .
\end{aligned}
$$

Our calculations replace $G_{0}$ by $G_{0}^{(L O)}$ of Eq. (9).

\section{SIMPLE MODELS AND ANALYTIC RESULTS}

One way to verify the $L O$ approximation is to show that it reproduces analytic results for previously published models. We consider the models of [9] and [13] in this section. In both of these models $p\left(g, g^{\prime}\right)$ is taken as a step function (the 0 temperature limit of our model):

$$
p\left(g, g^{\prime}\right)=\Theta\left(g+g^{\prime}-\mu\right) .
$$

The two models differ in their choice of $\rho(g)$, but the use of Eq. (16) allows one to obtain compact general expressions for the generating functions $\widehat{G}_{0}(z, g), \widehat{G}_{0}(k, g), p_{k}$ and $c(k)$. We present these first and discuss specific details of the individual models in separate sub-sections.

The use of Eq. (16) in Eq. (2) yields

$$
\ln \widehat{G}_{0}(z, g)=N\left[\Theta(\mu-g) \int_{\mu-g}^{\infty} d g^{\prime} \rho\left(g^{\prime}\right)+\Theta(g-\mu)\right] \log (z)=N \bar{p}(g) \log (z),
$$

so that

$$
\widehat{G}_{0}(z, g)=z^{N \bar{p}(g)} .
$$


It is interesting to observe that Eq. (8) reduces to the above result. This is because powers of $p\left(g, g^{\prime}\right)^{m}=p\left(g, g^{\prime}\right)$ for Eq. (16), so that the integration appearing in Eq. (8) leads to an expression that is a function of $\bar{N}, z, \bar{p}$. Then the use of the binomial theorem allows the second term of Eq. (8) to be expressed as a summable power series in $\bar{p}$ which ultimately leads to the result Eq. (18).

If we follow [11] and treat $k$ as a continuous variable (which requires large values of $k$ ) we find

$$
\widehat{G}_{0}(k, g)=\delta(k-N \bar{p}(g))=\frac{\delta\left(g-g_{N}(k)\right)}{N\left|\bar{p}^{\prime}\left(g_{N}(k)\right)\right|},
$$

where $g_{N}(k)$ is the solution of the equation

$$
k=N \bar{p}(g) .
$$

Note that for $k=N, g_{N}(k)$ can take on any value greater than $\mu$. The result Eq. 19$)$ is the same as eq.(34) of [11], but written in a more compact form. The use of Eq. (19) in Eq. (13) and Eq. (14) yields the results

$$
\begin{aligned}
p_{k} & =\frac{\rho\left(g_{N}(k)\right)}{N\left|\bar{p}^{\prime}\left(g_{N}(k)\right)\right|} \\
\bar{c}(k) & =\frac{c\left(g_{N}(k)\right)}{N\left|\bar{p}^{\prime}\left(g_{N}(k)\right)\right|} .
\end{aligned}
$$

\section{A. Model of Caldarelli et al. [9]}

This model is defined by using $\rho(g)=\exp (-g)$, but we generalize to take the form

$$
\rho_{\lambda}(g)=\lambda \exp (-\lambda g) \text {. }
$$

Ref. [11] works out this model using their Green's function formalism. Our purpose here is to compare the results of our averaging approximation with their results. For this model the average interaction probability $\bar{p}(g)$ is given by

$$
\bar{p}(g)=\int_{0}^{\infty} d g^{\prime} \lambda \exp \left[-\lambda g^{\prime}\right] \Theta\left(g+g^{\prime}-\mu\right)=\Theta(g-\mu)+\Theta(\mu-g) \exp [-\lambda(\mu-g)] .
$$

Then our approximation Eq. (13) for the degree distribution $p_{k}$ is given by

$$
p_{k}=\left(\begin{array}{c}
N \\
k
\end{array}\right) \int_{0}^{\mu} d g \lambda \exp [-\lambda g] \exp [-k \lambda(\mu-g)](1-\exp [-\lambda(\mu-g)])^{N-k}
$$

Define the integration variable $t \equiv \exp [-\lambda(\mu-g)]$ so that

$$
\begin{aligned}
& p_{k}=\left(\begin{array}{c}
N \\
k
\end{array}\right) e^{-\lambda \mu} \int_{t_{0}}^{1} \frac{d t}{t^{2}} t^{k}(1-t)^{N-k}, \quad t_{0} \equiv e^{-\lambda \mu} \\
& p_{k>1}=\left(\begin{array}{c}
N \\
k
\end{array}\right) e^{-\lambda \mu}\left(\frac{\Gamma(N+1-k) \Gamma(k-1)}{\Gamma(N)}-B_{t_{0}}(k-1, N+1-k)\right), \\
& p_{k=1}=N e^{-\lambda \mu} \frac{\left(1-t_{0}\right)^{N}}{N}{ }_{2} F_{1}\left(1, N ; N+1,1-t_{0}\right)
\end{aligned}
$$


where ${ }_{2} F_{1}$ is the confluent hypergeometric function and $B_{t_{0}}$ is the incomplete Beta function (and with $t_{0}=1$ the Beta function):

$$
B_{z}(a, b) \equiv \int_{0}^{z} d t t^{a-1}(1-t)^{b-1}, B_{1}(a, b)=B(a, b)
$$

Consider the case

$$
1<k, \quad \lambda \mu \approx 10
$$

(the latter is typical of our biological model) so that the second term of Eq. (28) can be neglected. Evaluating the remaining gamma functions gives

$$
p_{k}=e^{-\lambda \mu} \frac{N}{k(k-1)} \text {. }
$$

Ref. [11] computes the degree distribution for this model in analytic manner, using the approximation Eq. (19) in which $k$ is treated as a continuous variable and therefore "is expected to perform poorly for small values of $k$ ". The result of [11] $\left(p_{k}^{B P S}\right)$ is

$$
p_{k}^{B P S}=e^{-\lambda \mu} \frac{N}{k^{2}}+e^{-\lambda \mu} \delta(k-N)
$$

which corresponds to agreement (for $k \neq N)$ within the stated domain of accuracy of Ref. [11]. The confluence of Eq. (32) and Eq. (33) provides a verification of the accuracy of the averaging approximation.

The results for $k=N$ seem to disagree, so we examine this more closely. Use Eq. (18) directly to obtain the generating function $G_{0}(z)$ as $G_{0}(z)=\int d g \rho(g) z^{N \bar{p}(g)}$. One obtains a result $z^{N}$ for all values of $g(g>\mu)$ such that $\bar{p}(g)=1$. Using this generating function yields the result

$$
p_{k=N}=\int d g \rho(g) \Theta(g-\mu) .
$$

The specific value of the integral depends on the choice of $\rho(g)$, but the result is a finite number for any choice of $\rho(g)$ that satisfies the normalization condition that its integral over its domain is unity. Thus we believe that the correct result of using the propagator (eq $(34)$ of [11] in their eq(11)) is

$$
p_{k}^{B P S}=e^{-\lambda \mu} \frac{N}{k^{2}}
$$

instead of Eq. (33), which is in agreement with our result.

Our approximation works very well in reproducing the computed clustering coefficient of [11. In particular, we evaluate $c(g)$ of Eq. 115 to find that

$$
\bar{c}(k)=\frac{1}{p_{k}}\left(\int_{0}^{\mu / 2} \exp (-g) G_{0}^{(L O)}(k, g)+\int_{\mu / 2}^{\mu} \exp (-g) G_{0}^{(L O)}(k, g)(2 g-\mu+1)\right) .
$$

Numerical evaluation of this approximate expression accurately reproduces the result of Fig. 3 of Ref. [1]. Thus our mean field approximation is accurate for both our model[12] and the model of Ref. [9], 
TABLE I: Parameters obtained in Ref. [12]

\begin{tabular}{|c|c|c|c|}
\hline Species & $\mathrm{N}$ & $\lambda$ & $\mu$ \\
\hline H. pylori & 732 & 0.88 & 7.06 \\
P. falciparum & 1,310 & 0.93 & 7.77 \\
S. cerevisiae & 4,386 & 1.18 & 7.94 \\
C. elegans & 2,800 & 1.29 & 8.19 \\
D. melanogaster & 2,806 & 1.53 & 8.89 \\
Human [21] & 1,494 & 0.64 & 10.6 \\
Human [22] & 1,705 & 0.67 & 10.2 \\
\hline
\end{tabular}

\section{PROTEIN PROTEIN INTERACTION NETWORK-MODEL OF SHI ET AL. [12]}

Our principal application is to the the PIN of Ref. [12. This model is based on the concept of free energy of association. For a given pair of proteins the association free energy (in units of $R T$ ) is assumed to deviate from an average value a number contributed by both proteins additively as $g+g^{\prime}$. This is a unique approximation to first-order in $g$ and $g^{\prime}$. Thermodynamics and the assumption that the interaction probability is independent of concentration allows us to write

$$
p\left(g, g^{\prime}\right)=1 /\left(1+e^{\mu-g-g^{\prime}}\right)
$$

which reduces to a step function in the zero temperature limit, but otherwise provides a smooth function. Increasing the value of $\mu$ weakens the strength of interactions, and previous results [12] showed the existence of an evolutionary trend to weaker interactions in more complex organisms. The probability that a protein has a value of $g$ is given by the probability distribution

$$
\rho_{\lambda}(g)=\frac{\lambda}{e} e^{-\lambda g},-1 \leq \lambda g \leq+\infty,
$$

where the positive real value of $\lambda$ governs the fluctuations of $g$. We previously chose the speciesdependent values of $\lambda$ and $\mu$ so as to reproduce measured degree distributions obtained using the yeast two-hybrid method $(\mathrm{Y} 2 \mathrm{H})$ that reports binary results for protein-protein binding under a controlled setting[16]. Those parameters are displayed in Table I. The impact of the parameters $\lambda$ and $\mu$ are explained in Ref. [12] and displayed in Fig. 3 of that reference. Increasing the value of $\lambda$ increases the causes a more rapid decrease of $p_{k}$-the slope of $p_{k}$ increases in magnitude. Increasing the value of $\mu$ decreases the magnitude of $p_{k}$ without altering the slope much for values of $k$ greater than about 10 . The ability to vary both the slope and magnitude of $p_{k}$ gives this model flexibility that allows us to describe the available degree distributions for different species.

We obtain an analytic form for the for $\bar{p}(g)$ Eq. (7) of this model. Given Eq. (38) and Eq. (37) we find an analytic result:

$$
\bar{p}(g, \lambda)={ }_{2} F_{1}(1, \lambda ; \lambda+1 ;-\exp (\mu-g))
$$




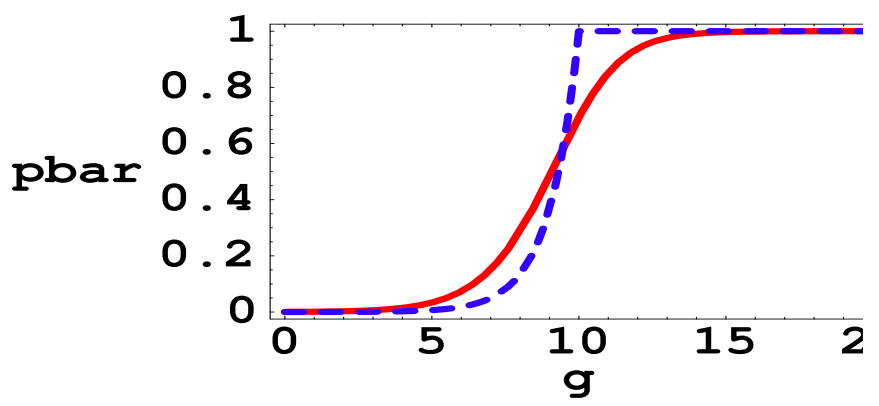

FIG. 1: (Color online) Average connection probability $\lambda=1, \mu=10$. Solid (Red): result of Eq. 40); dashed (blue) (containing the step function) result of Eq. 25). The approach to unity is smooth for Eq. 40.

where ${ }_{2} F_{1}$ is the confluent hypergeometric function. The special case $\lambda=1$ yields a closed form expression:

$$
\bar{p}_{1}(g)=e^{g-\mu} \ln \left(1+e^{\mu-g}\right) .
$$

A smooth average connection probability is obtained in contrast with the result of the sharp cutoff model Eq. (25). This shown in Fig. 1.

It is useful to define the variable

$$
\xi \equiv \exp (\mu-g)>0
$$

and note that an integral representation[14]

$$
{ }_{2} F_{1}(n, \lambda ; \lambda+1 ;-\xi)=\lambda \int_{0}^{1} d t t^{\lambda-1}(1+\xi t)^{-n}
$$

is convenient for numerical evaluations.

Knowledge of the propagator Eq. (9) allows us to compute the clustering coefficients of diverse species. The resulting degree distributions of $p_{k}$ (shown for the sake of completeness) and the newly computed clustering coefficients $\bar{c}(k)$ for yeast $S$. cerevisiae [17], worm C. elegans [18] and fruit fly D. melanogaster [19] are shown in Fig. 2. The parameters $\lambda$ and $\mu$ are 

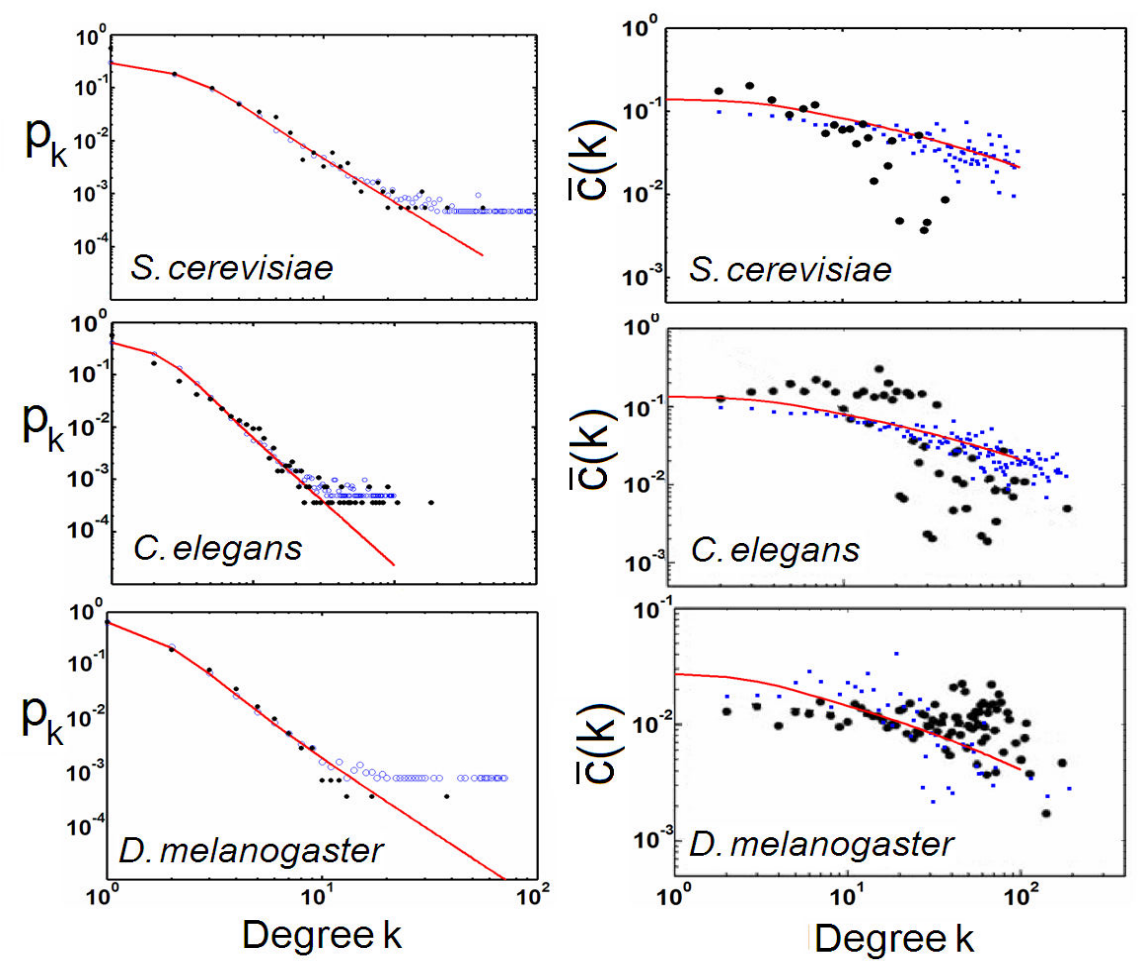

FIG. 2: (Color online) Degree distributions $p_{k}$ and clustering coefficients $\bar{C}(k)$ of diverse species. Degree distributions $p_{k}$ : The solid (red) curves are derived from the $L O$ theory. The black dots are the results of experimental data as referenced in the text. The small (blue) circles are the results of a numerical simulation using the procedure of [12]. Clustering coefficients $C \overline{(k)}$ : The solid (red) curves are derived from the $L O$ theory. The small (blue) dots are the results of a numerical simulation using the procedure of [12] and the heavy (black) dots represent the measured data.

those of [12], so the calculations of the clustering coefficients represent an independent major new prediction of our model. Results of numerical simulations and our analytic procedure are presented. The excellent agreement between the two methods verifies the $L O$ approximation. More importantly, the agreement between our calculations and the measured clustering coefficients is generally very good, so our model survives a very significant test. This bolsters the notion that the properties of a PIN are determined by a distribution of free energy. The clustering coefficient for yeast drops rapidly for large values of $k$ (where statistics are poor), a feature not contained in our model.

It is worthwhile to compare our model with that of [13. That work chooses a Gaussian form of $\rho(g)$, based on hydrophobicity, a step function form of $p\left(g, g^{\prime}\right)$, and is applied only to yeast. We found [12] that $p_{k}$ of [13] is scale free only for a narrow range of parameters, and we could not reproduce the data for diverse species using that model.

The human interactome is of special interest. Fig. 3A shows the human degree distributions computed with two sets of parameters, one from Ref. [12] (Table I) and the other using values of $\lambda=0.94, \mu=8.27$ shown in the caption. The degree distributions are essentially identical, so only one curve can be shown. Each is approximately of a power law form and each describes 
the measured degree distribution very well[20]. Calculations of degree correlations allows one to distinguish the two parameter sets. Figure $3 \mathrm{~B}$ shows that the cluster coefficients differ by a factor of two. We find that $\bar{c}(k)$ decreases substantially as $\lambda$ increases. The increase in $\lambda$ reduces the allowed spread in the value of $g$ and reduces the value of integrand of Eq. (14). It is interesting to note that the two existing measurements of the human $\bar{c}(k)$ differ by a factor of about an order of magnitude with the measurements of Ref.[22] obtaining much smaller values than those of [21]. The results of [21] are closer to our computed $\bar{c}(k)$ results for $\lambda=0.94, \mu=8.3$. In contrast with the results for other species, our $\bar{c}(k)$ lie significantly above the data. However, the two data sets disagree substantially (by a factor of as much as 100 for certain values of $k$ ) and both show a clustering coefficient that is generally significantly smaller than that of the other species. Several possibilities may account for the discrepancies between these two measurements of $\bar{c}(k)$ in humans and also for the differences between our model predictions and the experimental results. i) The human studies sample a limited subset of links of the complete network and this could bias the results. ii) The human protein subsets used in the two studies differ. iii) The human interactome is truly less connected than that of other species. This demonstrates the importance of measuring degree correlations to determine the underlying properties of the network. The current model and these considerations suggest the need for better design of future PIN studies that will not only include other species, but also comparisons between the PINs of different organs of a given species. Furthermore, comparisons between normal and malignant tissues could also be very fruitful.

\section{SUMMARY AND DISCUSSION}

In summary, this work provides a method to obtain the properties of hidden variable network models. The use of the approximation Eq. (7), used to obtain the propagator Eq. (9), provides an excellent numerical approximation to exact results for the models considered here. If necessary, the method can be systematically improved through the calculation of higher order corrections. Our principal example is the PIN of Ref. [12]. Not only does the use of Eq. (9) provide an accurate numerical result, but the model correctly predicts the clustering coefficients of most species. For the human interactome, two different parameter

sets yield nearly the same degree distribution but very different clustering coefficients, showing the importance of measuring degree correlations to determine the underlying nature of the network.

This work was supported in part by National Institutes of Health Grants GM45134 and DK45978 (to K.B.). We thank the authors of Refs. [21, 22] for providing tables of their data.

[1] S. H. Strogatz, Nature (London) 410,268 (2001).

[2] R. Albert and A.-L.Barabási, Rev. Mod. Phys. 74,47 (2002)

[3] M. E. J. Newman, SIAM Rev. 45, 167 (2003).

[4] R. Pastor-Satorras, A. Vázquez, and A. Vespignani, Phys. Rev. Lett. 87, 258701 (2001).

[5] A. Vázquez, R. Pastor-Satorras, and A. Vespignani, Phys. Rev. E 65, 066130 (2002) 

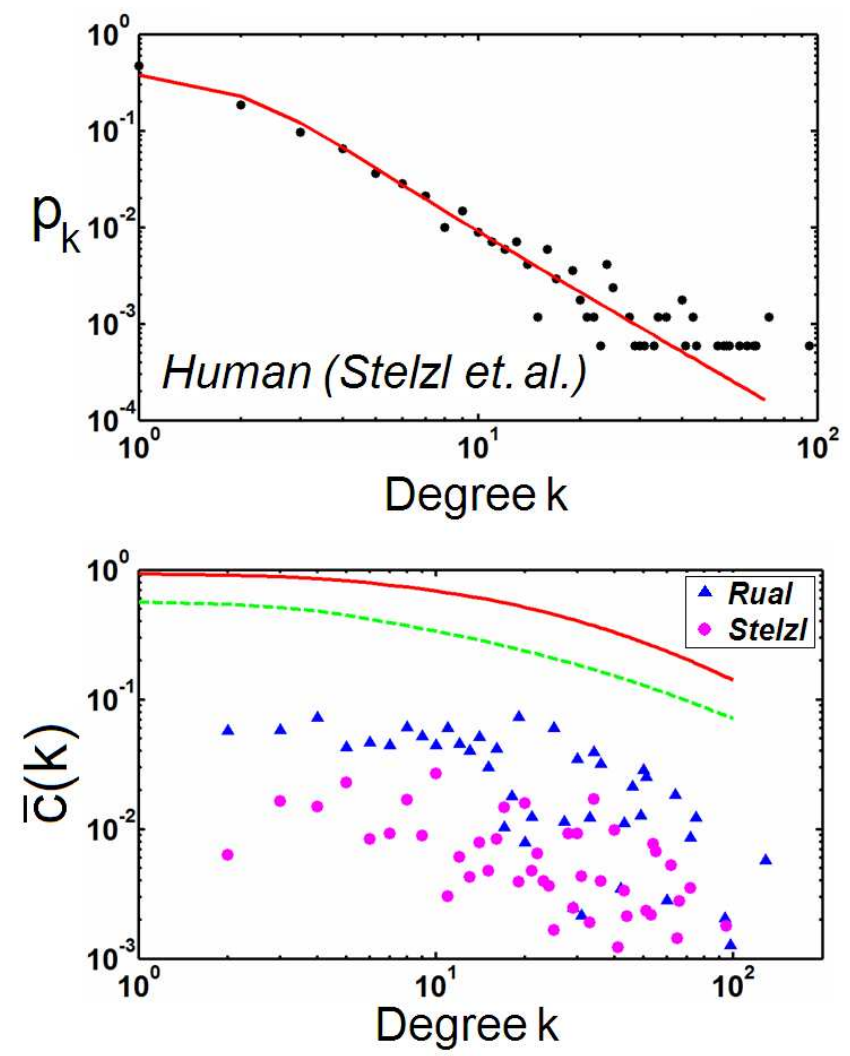

FIG. 3: (Color online) Human degree distribution $p_{k}$; the solid (red) curve is obtained using both set $\mathrm{A} \lambda=0.67, \mu=10.6$ and set $\mathrm{B} \lambda=0.94, \mu=8.3$. The black dots represent the experimental data. The data set is that of [21], but nearly identical data is obtained from [22]. Human cluster coefficient $\bar{c}(k)$ : The solid (red) curve is computed using set $\mathrm{A} \lambda=0.67, \mu=10.6$ and the dashed (green) using set B $\lambda=0.94, \mu=8.3$. Measured human clustering coefficients are from [21] triangles (blue) and [22] heavy dots (pink).

[6] M. E. J. Newman, Phys. Rev. Lett. 89, 208701,2002.

[7] M. E. J. Newman, Phys. Rev. E 67, 026126 (2003).

[8] B. Alberts et al. , The Cell, (Garland Science, New York 2002).

[9] G. Caldarelli, A. Capocci, P.DeLosRios, and M. A. Muñoz, Phys. Rev. Lett. 89, 258702 (2002).

[10] B. Söderberg, Phys. Rev. E 66, 066121 (2002).

[11] M. Boguñá and R. Pastor-Satorras, Phys. Rev. E 68, 036112 (2003).

[12] Yi Y. Shi, G.A. Miller, H. Qian, and K. Bomsztyk, Proc. Nat. Acad. Sci. 103, 11527 (2006).

[13] E.J. Deeds, O. Ashenberg, and E.I. Shakhonovich, Proc. Nat. Acad. Sci. 103, 311 (2006).

[14] M. Abramowitz and I. A. Stegun, Handbook of Mathematical Functions, (Dover, New York 1970).

[15] S. Abe and S. Thurner, Phys. Rev. E 72, 036102 (2005); S. Abe and S. Thurner, Int. J. Mod. 
Phys. C 17, 1303 (2006).

[16] S. Fields and S. Song, Nature 340, 245 (1989).

[17] http://www.nd.edu/ networks/resources/protein/bo.dat.qz

[18] S. Li, et al., Science 303, 540 (2004).

[19] L. Giot et al., Science 302, 1727 (2003).

[20] The value of $p_{0}$ is a testable result of our model, even though experimentalists do not measure this quantity. The predicted number of proteins with no interactions is $p_{0} N$, where the value of $N$ is given in Table I. The experimentalists conventionally normalize their distributions as $\sum_{k=1}^{\infty} p_{k}=1$, so we multiply our computed $p_{k}$ by a factor of $1 /\left(1-p_{0}\right)$ so that the computed $\operatorname{sum} \sum_{k=1}^{\infty} p_{k}$ is unity.

[21] J.F. Rual, et al. Nature 437, 1173 (2005)

[22] U. Stelzl, el al. Cell 122, 957 (2005) 